\title{
Loss of Quantum Efficiency in Green Light Emitting Diode Dies at Low Temperature
}

\author{
Yufeng $\mathrm{Li}^{1,2}$, Wei Zhao ${ }^{1,2}$, Yong Xia ${ }^{1,2}$, Mingwei Zhu ${ }^{1,2}$, Jayantha Senawiratne ${ }^{1,2}$, \\ Theeradetch Detchprohm ${ }^{1,2}$, E. Fred Schubert ${ }^{1,3}$, and Christian M Wetzel ${ }^{1,2}$ \\ ${ }^{1}$ Future Chips Constellation, Rensselaer Polytechnic Institute, 110 Eighth Street, Troy, \\ NY, 12180 \\ ${ }^{2}$ Department of Physics, Applied Physics, and Astronomy, Rensselaer Polytechnic \\ Institute, 110 Eighth Street, Troy, NY, 12180 \\ ${ }^{3}$ Department of Electrical, Computer and Systems Engineering, Rensselaer Polytechnic \\ Institute, 110 Eighth Street, Troy, NY, 12180
}

The electroluminescence, photoluminescence and cathodoluminescence of $\mathrm{GaInN} / \mathrm{GaN}$ multiple quantum well light emitting diode dies are analyzed at variable low temperature. Three dies of nominally identical structure but strongly different RT performance from $510 \mathrm{~nm}$ to $525 \mathrm{~nm}$ have been studied. The electroluminescence peak energy exhibits a blue shift from RT to $158 \mathrm{~K}$ followed by a red shift for lower temperature. In the same low-temperature range, a secondary emission peak appears near $390 \mathrm{~nm}$ $(3.18 \mathrm{eV})$ that resembles a donor-acceptor pair transition from GaN. Depth profiling spectroscopy of this transition at $77 \mathrm{~K}$ reveals its location either in the unintentionally doped quantum barriers or within the $\mathrm{n}$-GaN layer, rather than the commonly believed $\mathrm{Mg}$ doped p-type GaN layers. The external quantum efficiency of each die increases as the temperature is lowered. A maximum is reached for all near $158 \mathrm{~K}$ while for lower temperature as low as $7.7 \mathrm{~K}$, the efficiency continuously drops. The pronounced efficiency maximum is tentatively assigned to a combination of temperature dependent mobility and shift of the actual pn-junction location.

Keywords: GaInN/GaN, green LED, external quantum efficiency, low temperature, donor-acceptor transition

\section{Introduction}

High-brightness, reliable and efficient pure green light emitting diodes (LEDs) are of high economic value. However, when the emission wavelength of a multiple quantum well (MQW) LED device moves into the green region (beyond $500 \mathrm{~nm}$ ) the quantum efficiency drops sharply. The radiative recombination processes in group-III nitride heterostructures remain the subject of continued investigation in order to increase performance of high brightness LEDs. The external quantum efficiency (EQE) shows a strong dependence on the injection current and temperature. For typical operation current, EQE is only a fraction of its maximum value at low current. A detailed spectroscopic analysis is therefore warranted to identify the limiting mechanism and to enhance performance in next generation solid-state lighting. Here we spectrally analyze the electroluminescence (EL) and EQE as a function of variable low temperature in three dies of nominally identical structure but very different RT output power near $520 \mathrm{~nm}$.

\section{Experimental}

Pseudomorphic GaInN/GaN MQW structures of nominally identical structure have been grown by metal-organic vapor-phase epitaxy on c-plane sapphire substrates using the technique of low-temperature deposited buffer layers.[1] The carrier concentration of n-type GaN films was controlled by Si doping to nearly $1 \times 10^{19} \mathrm{~cm}^{-3}$. Five periods of GaInN/GaN QW with $3 \mathrm{~nm}$ well width have been grown thereafter. A $0.03 \mu \mathrm{m}$ p-type $\mathrm{AlGaN}$ cladding layer covers the QWs, followed by $0.2 \mu \mathrm{m}$ p-doped $\mathrm{GaN}$ with $\mathrm{Mg}$ 
concentration of $1 \times 10^{19} \mathrm{~cm}^{-3}$. Further details of the growth have been published elsewhere.[2] Individual dies were prepared by thinning of the substrate, die separation and mounting on $\mathrm{Cu}$ heat sinks. EL as a function of current from $0.1 \mathrm{~mA}$ to $30 \mathrm{~mA}$ was spectrally resolved in a $30-\mathrm{cm}$ monochromator and detected using a spectrally calibrated charge-coupled device. Total emission power was scaled to that measured in a calibrated integrating sphere. Different sample temperatures from RT to $7.7 \mathrm{~K}$ were achieved with a liquid $\mathrm{He}$ cryostat. Temperature was measured at the $\mathrm{Cu}$ sample stage and may be somewhat higher on the sample chip under current operation.

From a set of 88 green measured LED dies, three samples were selected on the basis of their EQE behavior. Under RT sample A has the maximum EQE of $4.7 \%$ at $2 \mathrm{~mA}$ and EQE of $3.2 \%$ at operation current $30 \mathrm{~mA}$, sample B has a lower performance with maximum EQE of $2.9 \%$ at $6 \mathrm{~mA}$ and $2.1 \%$ at 30 $\mathrm{mA}$. Sample $\mathrm{C}$ has an EQE that increased with current and reaches its maximum at $2.2 \%$ at $30 \mathrm{~mA}$. Further details have been given elsewhere.[3] All samples emit near $520 \mathrm{~nm}$ and serve as characteristic representatives of the full 88-sample set.[4] The following discussion will focus on the best performing sample A. Spectrally resolved noise properties of samples fabricated from the identical wafers have been reported recently.[5]

\section{Results and Discussion}

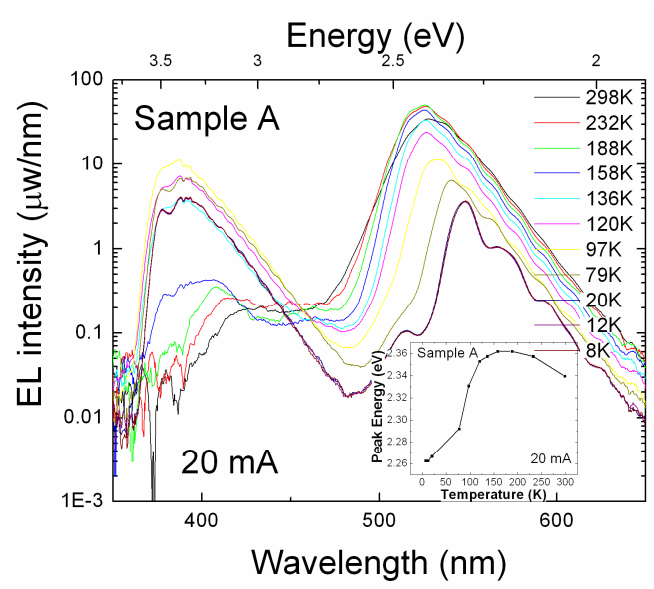

Fig. 1 Emission of LED A under different low temperature with an injection current of $20 \mathrm{~mA}$. The inset is the peak emission energy shift with temperature.

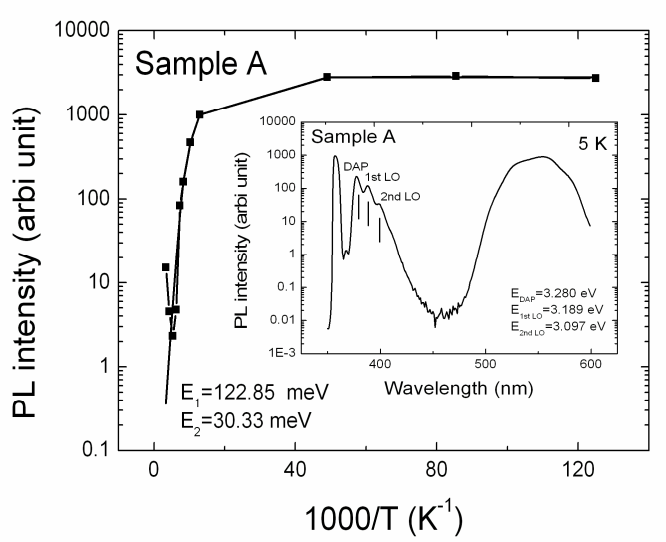

Fig. 2 PL Intensity of DAP as a function of inverse temperature. The inset shows the PL spectrum at $5 \mathrm{~K}$.

Fig. 1 shows the intensity-calibrated spectra of sample $\mathrm{A}$ at different temperatures with injection current of $20 \mathrm{~mA}$. The peak emission wavelength of the QW shifts to the blue from RT to $136 \mathrm{~K}$ and then to the red from $136 \mathrm{~K}$ to $7.7 \mathrm{~K}$. The blue-shift of about $20 \mathrm{meV}$ can be described by the Varshni equation of the temperature dependent band gap energy, while the red-shift of about $70 \mathrm{meV}$ at lower temperature resembles that of a band-tail effect.[6] A secondary emission appears around $390 \mathrm{~nm}(3.17 \mathrm{eV})$ at $136 \mathrm{~K}$ moving to $378 \mathrm{~nm}(3.28 \mathrm{eV})$ at $7.7 \mathrm{~K}$ accompanied by two replica $91 \mathrm{meV}$ and $182 \mathrm{meV}$ lower in energy. From the characteristic emission energy and double peak separation, we assign it to shallow donor-acceptor pair (DAP) recombination with LO-phonon replica.[7] This is also true for photoluminescence $(\mathrm{PL})$ under $7.7 \mathrm{~K}$. Fig. 2 shows the intensity of the DAP transition in PL as a function of inverse temperature from $297 \mathrm{~K}$ to $7.7 \mathrm{~K}$. Activation energies deduced by fitting the data with an Arrhenius model are $30 \mathrm{meV}$ and $123 \mathrm{meV}$. The candidate shallow donor might be $\mathrm{Si}\left(\mathrm{E}_{\mathrm{A}}=0.12-0.20\right.$ $\mathrm{eV})$ or $\mathrm{C}\left(\mathrm{E}_{\mathrm{A}}=0.11-0.21 \mathrm{eV}\right)$ on $\mathrm{Ga}$ site.[8] The smaller energy is responsible for the intensity variation in the low-temperature range and corresponds to the energy that ionizes the shallow donor. The 
corresponding cross section is four orders of magnitude smaller than that of the larger energy. The larger energy corresponds to the activation energy of the shallow acceptor. This ionization process induces an intensity reduction by two orders of magnitude from $158 \mathrm{~K}$ to RT. In order to associate the location of this DAP transition in the layer stack we performed depth profiling spectroscopy (Fig. 3). When the sample is cooled to $77 \mathrm{~K}$ by liquid nitrogen and investigated by cathodoluminescence (CL) with an acceleration voltage of $6 \mathrm{kV}$ (red curve), the QW starts to emit photons while no clear GaN near band-edge emission or DAP transition is observed. This shows that the DAP transition should not come from the p-type $\mathrm{GaN}$ above the QW. Instead it might either come from the n-type GaN or the unintentionally doped GaN quantum barrier or both.

By comparing the intensities of QW emission and DAP transition as the temperature drops in EL (injection current is $20 \mathrm{~mA}$ ) and PL (acceleration voltage is $12 \mathrm{kV}$ ) (Fig. 4) we find that the EL intensity experiences an s-shape evolution while the PL intensity changes monotonically as temperature drops until 7.7 K. The DAP transition appears in both EL and PL below $136 \mathrm{~K}$, however, the intensity shows a different dependence on temperature.

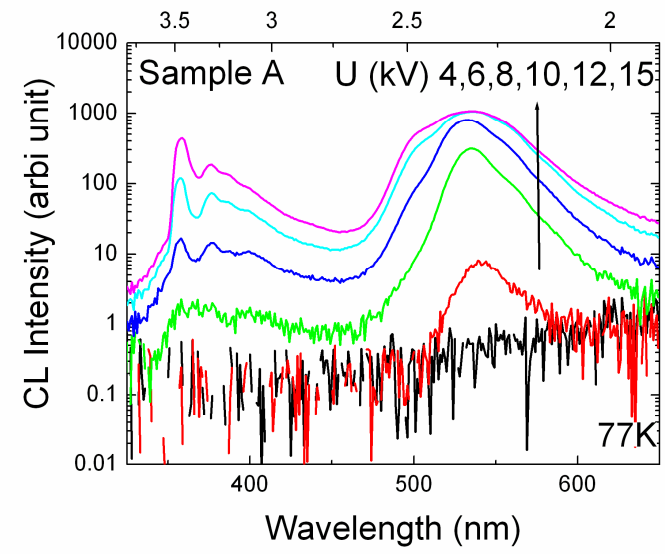

Fig. 3 CL spectrum for sample A under increasing acceleration voltage from $4 \mathrm{kV}$ to $15 \mathrm{kV}$ at $77 \mathrm{~K}$.

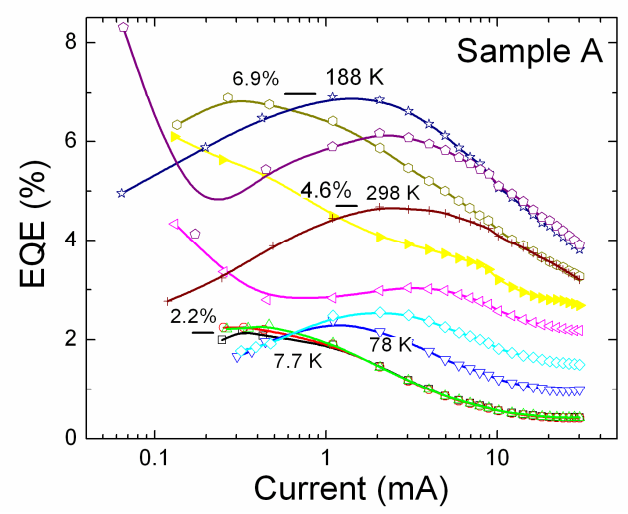

Fig. 5 EQE of sample A as a function of current from $7.7 \mathrm{~K}$ to RT.

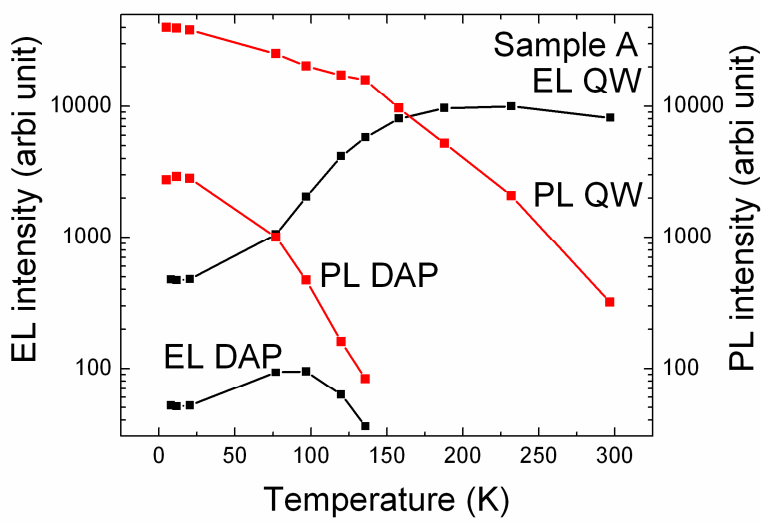

Fig. 4 EL (20 mA), PL intensity of QW and DAP emission as a function of temperature for sample A.

EQE for sample A as a function of current under variable low temperature is shown in Fig. 5. Basically the EQE behaves non-monotonically with temperature. The highest EQE increases from $4.6 \%$ at RT to $6.9 \%$ at $188 \mathrm{~K}$ and then drops to $2.2 \%$ at $7.7 \mathrm{~K}$. The current where EQE reaches its maximum also shifts to smaller values as the temperature decreases.

The reason that EQE increases and then decreases with decreasing temperature is diversified. The PL intensity indicates that the radiative recombination rate in quantum well goes up as then temperature is lower down. However it can not be observed in EL spectrum. The reason we propose is pn-junction shifts it position from $\mathrm{p}$-side to $\mathrm{n}$-side at low temperature, thus produce a loss of carrier recombination population. This 
mechanism competes with the enhanced radiative recombination rate and result the novel behavior of EQE in EL.

The pn- junction changes its location within the active region as dopants freeze out with lower temperature. Donors and acceptors freeze out at a different rate and the pn-junction therefore should easily move from the $\mathrm{n}$-side to the $\mathrm{p}$-side as the temperature is lowered. However, the depth profiling spectroscopy suggests that the pn-junction actually is moving towards n-side where DAP transitions happen. This necessarily detunes the charge balance from its optimum point of radiative recombination. Such a mechanism competes with the increasing of radiative recombination rate resulting from localization by a larger factor and thus results in the drop of efficiency below a certain temperature. For PL, the electrons and holes are generated by optical excitation inside the QW, so that there are much less carriers that are to recombine non-radiatively outside the active region as is the case in EL. Thus the PL intensity may show a monotonic increase with decreasing temperature.

On the other hand, EL most directly includes the charge carrier transport properties of the underlying diode. In its vertical transport scheme, electrical injection relies on majority carriers of electrons in the n-layer and holes in the p-layers. Considering mobility alone as determined in lateral transport geometry, electrons typically have their maximum mobility values at $158 \mathrm{~K}$,[9] while hole mobility only peaks at higher values as $200 \mathrm{~K}$.[10] Under lower temperature, mobility is limited by impurity scattering, while at higher temperature it is lattice scattering which dominates.[11] Charge transport includes the product of mobility and carrier concentration. Considering the temperature dependence of free electron and free hole concentrations, both maxima must be shifted to higher temperature. From this rough analysis, only electrons could produce a maximum near $158 \mathrm{~K}$, while regular hole transport should be strongly suppressed below such temperature. Overall transport in constant current mode, however, is not suppressed even at the lowest temperature because of local field emission, hole-trapping potential well and interface states [12] that are believed to maintain current flow in the c-plane GaInN/GaN LED at low temperature. Further more, the thermal conductivity along the c-axis of GaN reaches its maximum between $158 \mathrm{~K}$ and $188 \mathrm{~K}$.[13] This physical property might also contribute to the maximum efficiency at such temperature. Beyond experimental evidence, a clear association to a physical process, however, is not known. So at present we prioritize an interpretation in terms of mobility and recombination competition.

\section{Conclusions}

The luminescence of high performing green LEDs was characterized at variable low temperature in all three of PL, CL and EL. Besides the dominant emission from the QW at $520 \mathrm{~nm}$, the GaN bandedge luminescence and - at lower temperatures - a donor-acceptor pair transition are observed. Both, EL under constant current and PL intensity increase with lower temperatures although at different rates. The EL efficiency, analyzed in form of EQE, saturates near $150 \mathrm{~K}$ and decreases again for lower temperatures, while PL continues to increase. EL is still observed at temperatures as low as 7.7 $\mathrm{K}$. The decrease in EQE at low temperatures coincides with the appearance of the donor-acceptor pair recombination. From depth resolved $\mathrm{CL}$ at $77 \mathrm{~K}$ we find that this DAP recombination does not originate in the acceptor doped p-side. The reverting EQE behavior is attributed to a competition between pn-junction shift and low temperature enhanced radiative recombine rate and temperature dependent carrier mobility.

\section{Acknowledgments}

This work was supported by a DOE/NETL Solid-State Lighting Contract of Directed Research and a DOE/BES contract through Sandia National Labs.

\section{References}

[1] I. Akasaki, in Nitride Semiconductors, Mat. Res. Soc. Symp. Proc. Vol. 482 (Materials Research Society, Pittsburgh 1998 ). p. 1. 
[2] C. Wetzel, T. Salagaj, T. Detchprohm, P. Li, and J. S. Nelson, Appl. Phys. Lett. 85, 6, (2004).

[3] W. Zhao, Y. Li, T. Detchprohm, and C. Wetzel, Phys. Stat. Sol. (c) 4, 9, (2007).

[4] W. Zhao, Y. Li, Y. Xia, M. Zhu, T. Detchprohm, E.F. Schubert, and C. Wetzel, in "GaN, AlN, InN, and Related Materials,” Eds. M. Kuball, T.H. Myers, J.M. Redwing, T. Mukai, Proc. Mat. Res. Soc. Symp. Vol. 892, FF12.2, (2006).

[5] S.L. Rumyantsev, C. Wetzel, and M.S. Shur, J. Appl. Phys. 100, 084506, (2006).

[6] P.G. Eliseev, P. Perlin, J.Lee, and M. Osinski, Appl. Phys. Lett. 71, 569, (1997).

[7] K. Kim, J.G.Harrison, J. Vac. Sci. Techn. A, 21, 134, (2003).

[8] Bougrov V., Levinshtein M.E., Rumyantsev S.L., Zubrilov A., in Properties of Advanced SemiconductorMaterials GaN, AlN, InN, BN, SiC, SiGe. Eds. Levinshtein M.E., Rumyantsev S.L., Shur M.S., John Wiley \& Sons, Inc., New York, 1-30, (2001).

[9] M. Ilegems and H. C. Montgomery, J. Phys. Chem. Solid 34, 885, (1973).

[10] M. Kneissl, D.P. Bour, L. T. Romano, B.S. Krusor, M. McCluskey, W.Goetz, and N. M. Johnson, in: Proceedings of SPIE -- Volume 3279, Light-Emitting Diodes: Research, Manufacturing, and Applications II, San Jose, California, USA, 28, 70 (1998).

[11] O.Manasreh, M.G.Hill Semiconductor Heterojunctions and Nanostructures, Light-Emitting Diodes: Research, Manufacturing, and Applications II, New York, USA, 305-306 (2005).

[12] H. Masui, M.C. Schmidt, A. Chakraborty, S. Nakamura and S.P. DenBaars, Jpn. J. Appl. Phys. 45, 7661, (2006).

[13] E.K. Sichel, and J.L. Pankove, J. Phys. Chem. Solids 38, 330 (1997). 